2 Interference Effect of $\mathrm{H}_{2} \mathrm{O}$ on $\mathrm{Hg}^{0}$ Removal by Mercury

3 Sorbent in Oxyfuel-combustion Atmosphere

4 Hengyuan Ran a, Hui Wang a, *, Jingmao Wu ${ }^{\text {a }}$, Yiming Zhu a, *, Jianfei Wu ${ }^{\text {a }}$, Haotian Shen ${ }^{\mathrm{b}}$

5 a School of Energy and Power Engineering, Nanjing University of Science and Technology, Nanjing,

6 210094, China

$7 \quad{ }^{b}$ Nanjing Institute of Future Energy System, Institute of Engineering Thermodynamics, Chinese

8 Academy of Sciences, Nanjing, 210000, China

9

10

Corresponding author

12 *E-mail: wanghui22@njust.edu.cn. Tel/Fax: +86-25-84314965.

13 *E-mail: ymzhu@njust.edu.cn. Tel/Fax: 86+25-84314965.

\title{
Content includes:
}

17 Number of pagers: 3

18 Number of figures: 1

19 Number of tables: 1 


\begin{tabular}{|c|c|c|c|c|c|}
\hline Sample & $\begin{array}{c}\text { Specific } \\
\text { surface area } \\
\left(\mathrm{m}^{2} / \mathrm{g}\right)\end{array}$ & $\begin{array}{l}\text { Average } \\
\text { pore size } \\
\text { (nm) }\end{array}$ & $\begin{array}{l}\text { Total pore } \\
\text { volume } \\
\left(\mathrm{cm}^{3} / \mathrm{g}\right)\end{array}$ & $\begin{array}{c}\text { Micropore } \\
\text { specific surface } \\
\text { area }\left(\mathrm{m}^{2} / \mathrm{g}\right)\end{array}$ & $\begin{array}{l}\text { Micropore total } \\
\text { pore volume } \\
\qquad\left(\mathrm{cm}^{3} / \mathrm{g}\right)\end{array}$ \\
\hline Corn Straw Char & 59.9 & 1.398 & 0.031 & 51.7 & 0.026 \\
\hline $\begin{array}{l}\text { Modified Corn } \\
\text { Straw Char }\end{array}$ & 118.1 & 1.570 & 0.061 & 96.7 & 0.050 \\
\hline
\end{tabular}




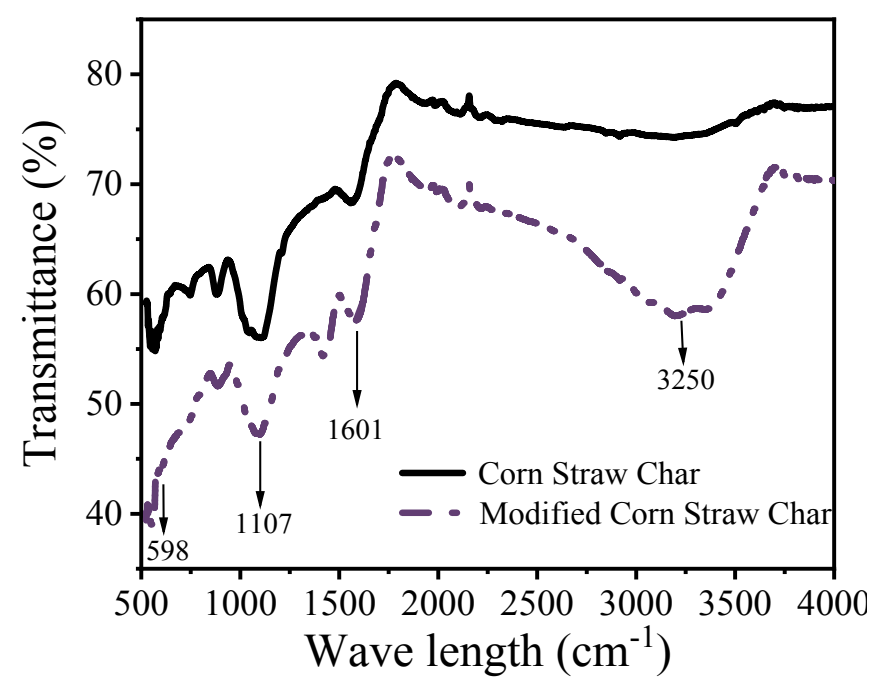

Fig S1. FTIR analysis of corn straw char before and after modification [1].

\section{Supporting References:}

42 [1]Shen HT, Wang H, Shen C, Wu JF, Zhu YM, Shi W, et al. Effect of atmosphere of $\mathrm{SO}_{2}$ coexisted

43 with oxidizing gas on mercury removal under oxy-fuel condition. Chemosphere 2020; 259: 127525. 